\title{
Novel weak decays in doubly strange systems
}

\author{
A. Parreño and A. Ramos \\ Departament d'Estructura i Constituents de la Matèria, Universitat de Barcelona, Diagonal 647, E-08028 Barcelona, Spain \\ C. Bennhold \\ Center for Nuclear Studies and Department of Physics, The George Washington University, Washington, D.C. 20052
}

(Received 22 June 2001; published 6 December 2001)

\begin{abstract}
The strangeness-changing $(\Delta S=1)$ weak baryon-baryon interaction is studied through the nonmesonic weak decay (NMD) of double- $\Lambda$ hypernuclei. Besides the usual nucleon-induced decay $\Lambda N \rightarrow N N$ we discuss novel hyperon-induced decay modes $\Lambda \Lambda \rightarrow \Lambda N$ and $\Lambda \Lambda \rightarrow \Sigma N$. These reactions provide unique access to the exotic $\Lambda \Lambda K$ and $\Lambda \Sigma K$ vertices that place new constraints on chiral pertubation theory ( $\chi \mathrm{PT})$ in the weak $\mathrm{SU}(3)$ sector. Within a meson-exchange framework, we use the pseudoscalar $\pi, \eta, K$ octet for the long-range part while parametrizing the short-range part through the vector mesons $\rho, \omega, K^{*}$. Realistic baryon-baryon forces for the $S=0,-1$, and -2 sectors account for the strong interaction in the initial and final states. For ${ }_{\Lambda \Lambda}^{6} \mathrm{He}$ the new hyperon-induced decay modes account for up to $4 \%$ of the total nonmesonic decay rate. Predictions are made for all possible nonmesonic decay modes.
\end{abstract}

DOI: 10.1103/PhysRevC.65.015205

\section{INTRODUCTION}

The production and weak decay properties of strangenessrich systems is of fundamental importance for our understanding of relativistic heavy-ion collisions and certain astrophysical phenomena, such as neutron stars. The simplest systems with strangeness, hypernuclei with one or two bound $\Lambda$ 's, have been used to study both the strong and the weak baryon-baryon $(B B)$ interaction in the $\mathrm{SU}(3)$ sector. Until now, hypernuclear weak decay represents the only source of information on the $|\Delta S|=1$ four-fermion interaction, where, in contrast to the weak $\Delta S=0 N N$ case, both the weak parity-conserving (PC) and parity-violating (PV) amplitudes can be studied.

In the absence of exact solutions to low-energy QCD, effective field-theory techniques based on chiral expansions have been fairly successful in the description of hadronic observables in the (nonstrange) SU(2) sector. The stability of the chiral expansion is less clear for the SU(3) sector, due to the significant amount of SU(3) symmetry breaking. A wellknown failure of $\mathrm{SU}(3)$ chiral perturbation theory has been the prediction [1] of the four PC $P$-wave amplitudes in the weak nonleptonic decays of octet baryons, $Y \rightarrow N \pi$, with $Y$ $=\Lambda, \Sigma$, or $\Xi$. Since large cancellations among tree-level amplitudes are held responsible for the problem with the weak $P$-wave octet amplitudes, it is imperative to assess whether this situation is universal within $\mathrm{SU}(3)$ chiral perturbation theory $(\chi \mathrm{PT})$ or limited to a few exceptional cases. However, other weak octet baryon-baryon-meson $(B B M)$ vertices can only be accessed through reactions that allow for the virtual exchange of mesons, such as the reactions $\Lambda N$ $\rightarrow N N$ and $\Lambda \Lambda \rightarrow \Lambda(\Sigma) N$. The process $\Lambda N \rightarrow N N$ has been extensively studied in an approach where the long-range part of this interaction is based on the exchange of the SU(3) pseudoscalar meson octet $(\pi, K$, and $\eta)$. The pseudoscalar baryon-baryon-meson vertices are considered fixed by experiment in the case of the pion, and by $\mathrm{SU}(3)$ chiral algebra for the $K$ and the $\eta$. Since the large momentum transfer in the reaction (typically $400 \mathrm{MeV} / \mathrm{c}$ ) leads to a mechanism
PACS number(s): 21.80.+a, 24.80.+y, 13.30.Eg, 13.75.Ev

where short-range effects must be included, they have been modelled either through the exchange of the vector meson octet [2-4] $\left(\rho, \omega\right.$, and $\left.K^{*}\right)$ or quark exchange [5]. The vector baryon-baryon-meson vertices are constrained by much weaker SU(6) considerations. The $\Lambda N \rightarrow N N$ process is then embedded in nuclear many-body matrix elements using either correlated Faddeev amplitudes in the case of few-body systems, hypernuclear shell model wave functions, or nuclear matter solutions within the local density approximation, depending on the mass number of the hypernucleus under investigation. While this description of hypernuclear weak decay is not as rigorous as effective field theory would require, it nevertheless has been reasonably successful in describing the available experimental data.

Since the late 1960s, the production and decay of single- $\Lambda$ hypernuclei have been studied experimentally in great detail, but only very few events involving doubly strange objects have been reported [6-12]. Double $\Lambda$ hypernuclei are produced via the $\left(K^{-}, K^{+}\right)$reaction at KEK (Japan) and BNL (USA), where a $\Lambda \Lambda$ hypernuclear fragment can be formed by $\Xi^{-}$capture on a nucleus. The FINUDA experiment at DAФNE (Frascati, Italy) can produce double- $\Lambda$ hypernuclei by stopping slow $K^{-}$(coming from the $\Phi$ decay) into thin targets to obtain data with higher energy resolution. Studying the weak decay of those objects opens the door to a number of new exotic $\Lambda$-induced decay modes: $\Lambda \Lambda \rightarrow \Lambda n$ and $\Lambda \Lambda$ $\rightarrow \Sigma N$. Both of these decays would involve hyperons in the final state and should be distinguishable from the ordinary $\Lambda N \rightarrow N N$ mode. The $\Lambda \Lambda \rightarrow \Lambda n$ channel is especially intriguing since the dominant pion exchange is forbidden, thus forcing this reaction to occur mostly through kaon exchange. One would therefore gain access to the weak $\Lambda \Lambda \mathrm{K}$ and $\Lambda \Sigma \mathrm{K}$ vertices.

In this paper, we extend previous weak decay calculations of single- $\Lambda$ hypernuclei into the $S=-2$ sector, thus exploring the power of the $\Lambda \Lambda \rightarrow \Lambda(\Sigma) N$ process to shed light on the novel weak vertices. In order to take into account the effects of the strong interaction between the baryons, correlated wave functions are obtained from a $G$-matrix calcula- 
tion for the initial $\Lambda N$ and $\Lambda \Lambda$ states, while a $T$-matrix equation is solved for the final $N N$ and $Y N$ states using the Nijmegen interaction models [13], in particular the NSC97f one.

\section{DECAY RATE}

In the weak nonmesonic decay of double- $\Lambda$ hypernuclei, new hyperon-induced mechanisms, the $\Lambda \Lambda \rightarrow \Lambda N$ and the $\Lambda \Lambda \rightarrow \Sigma N$ transitions (denoted as $\Lambda \Lambda \rightarrow Y N$ throughout the text) become possible in addition to the dominant $\Lambda N$ $\rightarrow N N$ decay mode. Assuming the initial hypernucleus to be at rest, the nonmesonic decay (NMD) rate is given by

$$
\begin{aligned}
\Gamma_{\mathrm{nm}}= & \int \frac{d^{3} k_{1}}{(2 \pi)^{3}} \int \frac{d^{3} k_{2}}{(2 \pi)^{3}} \sum_{\substack{M_{,}\{R\} \\
\{1\}\{2\}}}(2 \pi) \delta\left(M_{H}-E_{R}-E_{1}-E_{2}\right) \\
& \times \frac{1}{(2 J+1)}\left|\mathcal{M}_{i \rightarrow f}\right|^{2}
\end{aligned}
$$

where the quantities $M_{H}, E_{R}, E_{1}$ and $E_{2}$ are the mass of the hypernucleus, the energy of the residual $(A-2)$-particle system, and the total asymptotic energies of the emitted baryons, respectively. The integration variables $\vec{k}_{1}$ and $\vec{k}_{2}$ stand for the momenta of the two baryons in the final state. In the expression above, the momentum-conserving delta function has been used to integrate over the momentum of the residual nucleus. The sum, together with the factor $1 /(2 J+1)$, indicates an average over the initial hypernucleus spin projections, $M_{I}$, and a sum over all quantum numbers of the residual $(A-2)$-particle system, $\{R\}$, as well as the spin and isospin projections of the emitted particles, $\{1\}$ and $\{2\}$. The total nonmesonic decay rate can be written as

$$
\Gamma_{\mathrm{nm}}=\Gamma_{N N}+\Gamma_{Y N},
$$

where the rate corresponding to $\Lambda N \rightarrow N N$ transitions

$$
\Gamma_{N N}=\Gamma_{n}+\Gamma_{p},
$$

is divided into a neutron-induced rate, $\Gamma_{n}: \Lambda n \rightarrow n n$ and a proton-induced one, $\Gamma_{p}: \Lambda p \rightarrow n p$, while the $\Lambda$-induced transitions $\Lambda \Lambda \rightarrow Y N$ give rise to $\Lambda n, \Sigma^{0} n$, and $\Sigma^{-} p$ final states; hence

$$
\Gamma_{Y N}=\Gamma_{\Lambda n}+\Gamma_{\Sigma 0_{n}}+\Gamma_{\Sigma-p}
$$

Note that while the individual rates have been written above as exclusive observables, in principle, chargeexchange final-state interactions (FSI) with the residual nucleus obscures a clean experimental discrimination of these channels on the basis of the charge of the emitted particles. Monte Carlo intranuclear cascade models are necessary [14] to extract the partial weak decay rates of hypernuclei from experiment [15]. The impossibility of measuring the partial rates directly from the charge of the emitted particles was also shown in the case of the weak decay of the hypertriton [16], a system where the strong interaction effects can be treated exactly using Fadeev equations with a realistic baryon-baryon potential.
In order to draw conclusions regarding the weak dynamics, one has to write the hypernuclear transition amplitude, $\mathcal{M}_{i \rightarrow f}$, in terms of the elementary two-body transitions, $B_{1} B_{2} \rightarrow B_{3} B_{4}$, and nuclear structure details have to be treated with as few approximations and ambiguities as possible. We work in a shell-model framework; hence the $\Lambda$ hyperons and the nucleons are described by single-particle orbitals. In addition, we assume a weak coupling scheme by virtue of which the $\Lambda$ hyperons couple only to the ground state of the nuclear core. Therefore, in the case of the ${ }_{\Lambda \Lambda}^{6} \mathrm{He}$ hypernucleus studied here, with quantum numbers $J_{I}=M_{I}=0$, $T_{I}=M_{T_{I}}=0$, the state will be given by

$$
\left|{ }_{\Lambda \Lambda}^{6} \mathrm{He}\right\rangle_{T_{I}=M_{T_{I}}=0}^{J_{I}=M_{I}=0}=|\Lambda \Lambda\rangle_{T_{\Lambda}=M_{T_{\Lambda}}=0}^{J_{J}=M_{\Lambda}=0} \otimes\left|{ }^{4} \mathrm{He}\right\rangle_{T_{c}=M_{T_{c}}}^{J_{c}=M_{c}=0},
$$

where antisymmetry forces the two $\Lambda$ hyperons to be in a ${ }^{1} S_{0}$ state, since they are assumed to be in the lowest $s$ shell before the decay occurs. This is so because, in general, hypernuclei with $\Lambda$ 's in excited orbitals will rapidly decay into the ground state through electromagnetic or strong deexcitation processes, which are orders of magnitude faster than those mediated by the weak interaction. The single-particle orbitals for nucleons and $\Lambda$ 's are taken as harmonic oscillator states with parameters $b_{N}=1.4 \mathrm{fm}$ and $b_{\Lambda}=1.6 \mathrm{fm}$, respectively. The nucleon parameter is chosen to account for the ${ }^{4} \mathrm{He}$ form factor. The parameter for the $\Lambda$ wave function reproduces the Hartree-Fock (HF) probability of finding the two $\Lambda$ particles at relative distance $r$, obtained in Ref. [17] by adjusting their model parameters to the binding energies of the three observed double- $\Lambda$ hypernuclei, ${ }_{\Lambda \Lambda}^{6} \mathrm{He},{ }_{\Lambda \Lambda}^{10} \mathrm{~B}$, and ${ }_{\Lambda \Lambda}^{13} \mathrm{~B}[6-10]$.

To obtain the rate corresponding to the $\Lambda N \rightarrow N N$ transition, with the initial $N$ being $n\left(t_{N}=-1 / 2\right)$ or $p\left(t_{N}=1 / 2\right)$, we have to write the nonstrange nuclear core as one nucleon coupled to a three-particle system (with quantum numbers $J_{3}, M_{3}, T_{3}$, and $M_{T_{3}}$ ) and decouple one of the two $\Lambda$ 's in the cluster. Therefore, the initial $\Lambda N$ pair will convert into a final $N N$ pair with quantum numbers $\vec{k}_{1} s_{1} t_{1}$ and $\vec{k}_{2} s_{2} t_{2}$, leaving a residual four-particle system with quantum numbers $J_{R}, M_{R}, T_{R}, M_{T_{R}}$. Performing all the necessary decoupling and recoupling operations, we finally arrive at

$$
\begin{aligned}
\mathcal{M}_{\Lambda N \rightarrow N N}= & \left\langle\vec{k}_{1} s_{1} t_{1}, \vec{k}_{2} s_{2} t_{2} ;{ }_{\Lambda}^{4} A_{T_{R} M_{T_{R}} I_{R}}^{J_{R}}|\hat{O}|_{\Lambda \Lambda}^{6} \mathrm{He}\right\rangle\left|\frac{1}{2}-\frac{1}{2}\right\rangle_{\Lambda} \\
= & \sum_{S} \sum_{T} \sum_{m_{N}} \sum_{S_{0}}\left\langle T M_{T} \mid \frac{1}{2} t_{1}, \frac{1}{2} t_{2}\right\rangle \\
& \times\left\langle T M_{T} \mid \frac{1}{2}-\frac{1}{2}, \frac{1}{2} t_{N}\right\rangle\left\langle T_{3} M_{T_{3}}, \frac{1}{2} t_{N} \mid T_{c}=0 M_{T_{c}}=0\right\rangle \\
& \times\left\langle J_{3} M_{3}, \frac{1}{2} m_{N} \mid J_{c}=0 M_{c}=0\right\rangle \\
& \times\left\langle\frac{1}{2}\left(m_{N}-M_{S_{0}}\right), J_{3} M_{3} \mid J_{R} M_{R}\right\rangle\left\langle S M_{S} \mid \frac{1}{2} s_{1}, \frac{1}{2} s_{2}\right\rangle \\
& \times\left\langle\frac{1}{2}\left(M_{S_{0}}-m_{N}\right), \frac{1}{2}\left(m_{N}-M_{S_{0}}\right) \mid J_{\Lambda}=0 M_{\Lambda}=0\right\rangle \\
& \times\left\langle\frac{1}{2}\left(M_{S_{0}}-m_{N}\right), \frac{1}{2} m_{N} \mid S_{0} M_{S_{0}}\right\rangle\left\langle\vec{K} \mid \Psi_{\Lambda \mathrm{N}}^{\mathrm{CM}}\right\rangle \\
& \times\left\langle\vec{k}, S M_{S}, T M_{T}|\hat{O}| \Psi_{\Lambda \mathrm{N}}^{\mathrm{rel}}, S_{0} M_{S_{0}}, T M_{T}\right\rangle
\end{aligned}
$$




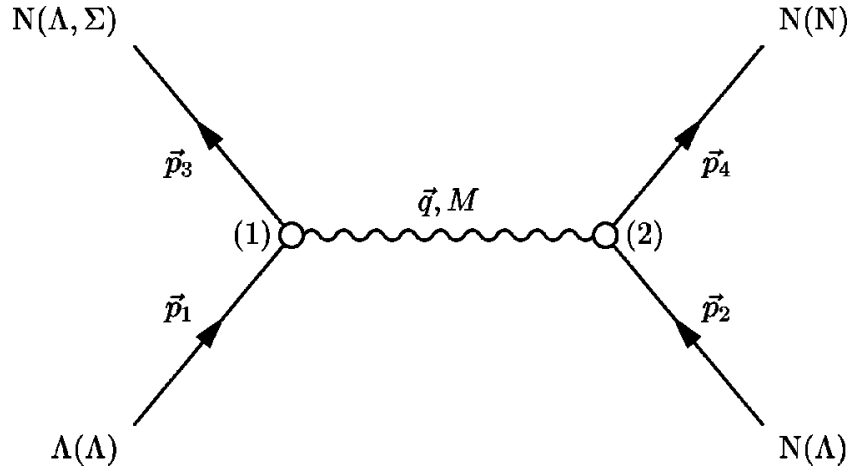

FIG. 1. Feynman diagram for the weak $\Lambda N \rightarrow N N$ and $\Lambda \Lambda$ $\rightarrow Y N$ ( $\Sigma N$ or $\Lambda n$ interaction).

where $T_{3}=1 / 2, \quad M_{T_{3}}=-t_{N}, \quad J_{3}=1 / 2, \quad M_{3}=-m_{N}, \quad M_{T_{R}}$ $=-t_{N}, M_{S_{0}}=-M_{R}, M_{S}=s_{1}+s_{2}$, and $M_{T}=t_{N}-1 / 2=t_{1}$ $+t_{2}$. We have assumed the $\Lambda$ at the weak vertex to behave as a $\left|\frac{1}{2}-\frac{1}{2}\right\rangle$ isospurion in order to naturally incorporate the experimentally observed $\Delta I=1 / 2$ rule. The momentum states $\vec{k}_{1}$ and $\vec{k}_{2}$ have been transformed to total $\left(\vec{K}=\vec{k}_{1}+\vec{k}_{2}\right)$ and relative $\left[\vec{k}=\left(\vec{k}_{1}-\vec{k}_{2}\right) / 2\right]$ momenta and the final two-nucleon state, $\left\langle\vec{k}, S M_{S}, T M_{T}\right|$, must be properly antisymmetrized, which means that the factor $\left[1-(-1)^{L+S+T}\right] / \sqrt{2}$ will appear when a decomposition in partial waves is performed for the outgoing plane wave.

For the $\Lambda \Lambda \rightarrow Y N$ transition we do not need to decouple one of the hyperons from the cluster, neither a nucleon from the core. The residual four-particle system, which coincides with the ${ }_{\Lambda}^{4} \mathrm{He}$ core in Eq. (5), contains no strangeness, while the final two-particle state contains one hyperon that can be either a $\Lambda\left(\left|Y t_{Y}\right\rangle=|00\rangle\right)$, a $\Sigma^{-}\left(\left|Y t_{Y}\right\rangle=|1-1\rangle\right)$, or a $\Sigma^{0}\left(\left|Y t_{Y}\right\rangle=|10\rangle\right)$. The $\Lambda \Lambda \rightarrow Y N$ hypernuclear amplitude is given by

$$
\begin{aligned}
\mathcal{M}_{\Lambda \Lambda \rightarrow Y N}= & \left\langle\vec{k}_{N} s_{N} t_{N}, \vec{k}_{Y} s_{Y} t_{Y} ;{ }^{4} \mathrm{He}|\hat{O}|{ }_{\Lambda \Lambda}^{6} \mathrm{He}\right\rangle\left|\frac{1}{2}-\frac{1}{2}\right\rangle_{\Lambda} \\
= & \sum_{S, M_{S}}\left\langle\frac{1}{2} s_{N}, \frac{1}{2} s_{Y} \mid S M_{S}\right\rangle\left\langle\frac{1}{2} t_{N}, Y t_{Y} \mid T M_{T}\right\rangle\left\langle\vec{K} \mid \Psi_{\Lambda \Lambda}^{\mathrm{CM}}\right\rangle \\
& \times\left\langle\vec{k}, S M_{S}, T M_{T}|\hat{O}| \Psi_{\Lambda \Lambda}^{\mathrm{rel}}, S_{0} M_{S_{0}}, T_{0} M_{T_{0}}\right\rangle,
\end{aligned}
$$

where $S_{0}=M_{S_{0}}=0, T=T_{0}=1 / 2$, and $M_{T}=M_{T_{0}}=-1 / 2$.

\section{THE MESON-EXCHANGE POTENTIAL}

In a meson-exchange description, the $\Lambda N \rightarrow N N$ and $\Lambda \Lambda \rightarrow Y N$ transitions are assumed to proceed via the exchange of virtual mesons belonging to the ground-state pseudoscalar and vector meson octets. The amplitude, which is schematically represented by the Feynman diagram depicted in Fig. 1, reads

$$
\begin{aligned}
\mathcal{M}_{\mathrm{M}}= & \int d^{4} x d^{4} y \bar{\Psi}_{p_{3}}(x) \Gamma_{1} \Psi_{p_{1}}(x) \Delta_{\mathrm{M}}(x-y) \\
& \times \bar{\Psi}_{p_{4}}(y) \Gamma_{2} \Psi_{p_{2}}(y)
\end{aligned}
$$

TABLE I. Pseudoscalar (PS) and vector (V) Hamiltonians entering Eq. (8) (in units of $G_{F} m_{\pi}^{2}=2.21 \times 10^{-7}$ ). $\Psi$ ( $\Phi$ ) stands for the baryon (meson) field.

\begin{tabular}{ccc}
\hline \hline & PS & $\mathrm{V}$ \\
\hline Strong & $\mathrm{i} g \bar{\Psi} \gamma_{5} \phi \Psi$ & $\bar{\Psi}\left[g^{\mathrm{V}} \gamma^{\mu}+\mathrm{i} \frac{g^{\mathrm{T}}}{2 M} \sigma^{\mu \nu} q_{\nu}\right] \phi \Psi$ \\
Weak & $\mathrm{i} \bar{\Psi}\left(A+B \gamma_{5}\right) \phi \Psi$ & $\bar{\Psi}\left[\alpha \gamma^{\mu}-\beta \mathrm{i} \frac{\sigma^{\mu \nu} q_{\nu}}{2 \bar{M}}+\varepsilon \gamma^{\mu} \gamma_{5}\right] \phi \Psi$ \\
\hline \hline
\end{tabular}

where $\Psi_{p}(x)=e^{-\mathrm{i} p x} u(p)$ is the free baryon field of positive energy, $\Gamma_{i}$ the Dirac operator characteristic of the baryonbaryon-meson $(B B M)$ vertex and $\Delta_{\mathrm{M}}(x-y)$ the meson propagator.

In Table I we show the strong and weak Hamiltonians for pseudoscalar (PS) and vector (V) mesons. The constants $A$, $B, \alpha, \beta$, and $\epsilon$ correspond to the weak couplings, while $g\left(g^{\mathrm{V}}, g^{\mathrm{T}}\right)$ represents the strong (vector, tensor) one.

The nonrelativistic reduction of this amplitude gives us the potential in momentum space [3], which for pseudoscalar mesons reads

$$
V_{\mathrm{ps}}(\vec{q})=-G_{F} m_{\pi}^{2} \frac{g}{2 M_{2}}\left(\hat{A}+\frac{\hat{B}}{2 M_{1}} \vec{\sigma}_{1} \cdot \vec{q}\right) \frac{\vec{\sigma}_{2} \cdot \vec{q}}{\vec{q}^{2}+\mu^{2}},
$$

where $G_{F} m_{\pi}^{2}=2.21 \times 10^{-7}$ is the Fermi weak constant, $\vec{q}$ is the momentum carried by the meson directed towards the strong vertex, $\mu$ the meson mass, and $M_{2}\left(M_{1}\right)$ is the average of the baryon masses at the strong (weak) vertex. For vector mesons the potential reads

$$
\begin{aligned}
V_{v}(\vec{q})= & G_{F} m_{\pi}^{2}\left(g^{\mathrm{V}} \hat{\alpha}-\frac{(\hat{\alpha}+\hat{\beta})\left(g^{\mathrm{V}}+g^{\mathrm{T}}\right)}{4 M_{1} M_{2}}\left(\vec{\sigma}_{1} \times \vec{q}\right)\left(\vec{\sigma}_{2} \times \vec{q}\right)\right. \\
& \left.-i \frac{\hat{\varepsilon}\left(g^{\mathrm{V}}+g^{\mathrm{T}}\right)}{2 M_{2}}\left(\vec{\sigma}_{1} \times \vec{\sigma}_{2}\right) \vec{q}\right) \frac{1}{\vec{q}^{2}+\mu^{2}}
\end{aligned}
$$

In Eqs. (9) and (10) the operators $\hat{A}, \hat{B}, \hat{\alpha}, \hat{\beta}$, and $\hat{\varepsilon}$ contain, apart from the weak coupling constants, the specific isospin dependence of the potential, which is $\vec{\tau}_{1} \vec{\tau}_{2}$ for the isovector $\pi$ and $\rho$ mesons, $\hat{1}$ for the isoscalar $\eta$ and $\omega$ mesons, and a combination of both operators for the isodoublet $K$ and $K^{*}$.

Reference [3] introduces a compact way to write the transition potential in $\vec{r}$ space:

$$
V(\vec{r})=\sum_{i} \sum_{\alpha} V_{\alpha}^{(i)}(\vec{r})=\sum_{i} \sum_{\alpha} V_{\alpha}^{(i)}(r) \hat{O}_{\alpha}(\hat{r}) \hat{I}_{\alpha}^{(i)},
$$

where the index $i$ runs over the different mesons exchanged ( $i=1, \ldots, 6$ represents $\left.\pi, \eta, K, \rho, \omega, K^{*}\right)$ and $\alpha$ over the different spin operators: central spin independent $(C)$, central spin dependent (SS), tensor (T), and parity violating (PV) (see Table II) 
TABLE II. Possible ${ }^{2 S+1} L_{J}$ channels present in the weak decay of ${ }_{\Lambda \Lambda}^{6}$ He. The notation used is the following: $L_{r}$ stands for the relative orbital momentum for the initial $\Lambda \Lambda$ or $\Lambda N$ pair, $L^{\prime}$ for the relative angular momentum of the two-particle state right after the weak transition occurs, and $L$ for the relative angular momentum of the final two-particle state after including the effects of the strong interaction (FSI). The symbols $\mathrm{C}, \mathrm{T}$, and PV stand for central, tensor, and parity violating.

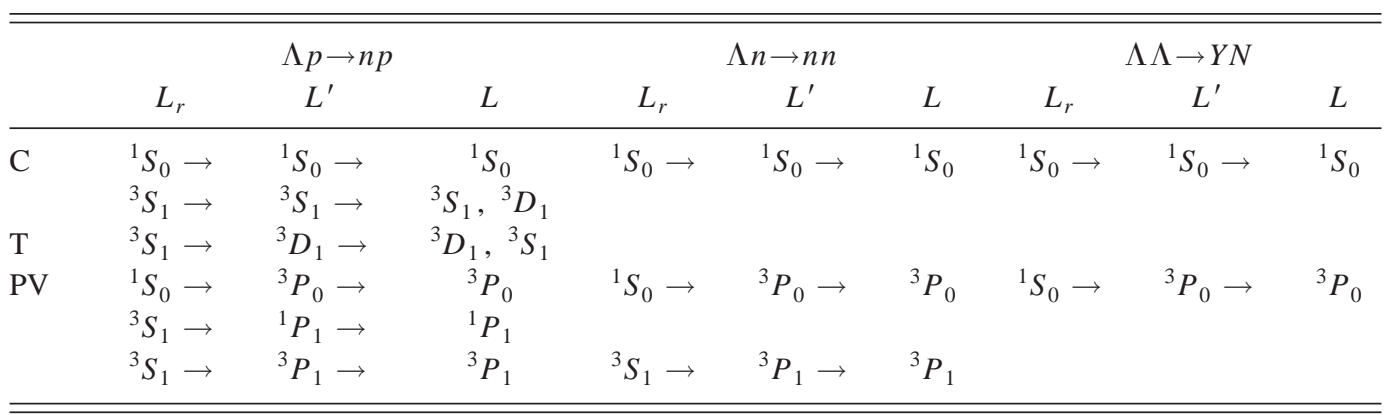

$$
\hat{O}_{\alpha}(\hat{r})= \begin{cases}\hat{1} & \mathrm{C} \text { (only for vector } \\ & \text { mesons), } \\ \vec{\sigma}_{1} \vec{\sigma}_{2} & \mathrm{SS}, \\ S_{12}(\hat{r})=3 \vec{\sigma}_{1} \hat{r} \vec{\sigma}_{2} \hat{r}-\vec{\sigma}_{1} \vec{\sigma}_{2} & \mathrm{~T}, \\ i \vec{\sigma}_{2} \hat{r} & \mathrm{PV} \text { (pseudoscalar } \\ & \text { mesons), } \\ {\left[\vec{\sigma}_{1} \times \vec{\sigma}_{2}\right] \hat{r}} & \mathrm{PV} \text { (vector mesons) }\end{cases}
$$

In order to account for the finite size and structure of the particles involved in the process, form factors (FF's) are included. In our previous calculations of the decay of single- $\Lambda$ hypernuclei [3] we used a monopole FF, $F\left(\vec{q}^{2}\right)=\left(\Lambda_{i}^{2}\right.$ $\left.-\mu^{2}\right) /\left(\Lambda_{i}^{2}+\vec{q}^{2}\right)$, at both the weak and strong vertices, with different cutoffs depending on the exchanged meson, $\Lambda_{i}$. Those cutoffs were taken from the Jülich $Y N$ interaction model [18]. The latest version of the Nijmegen potentials [13], which is used here, also gives meson-dependent cutoffs but uses an exponential $\mathrm{FF}$ at each vertex of the type $F\left(\vec{q}^{2}\right)=\exp \left(-\vec{q}^{2} / 2 \Lambda_{i}^{2}\right)$. Therefore, the results presented here are also obtained with the exponential-type FF, although, for technical reasons, it is matched to a function of the type $\tilde{\Lambda}_{i}^{2} /\left(\tilde{\Lambda}_{i}^{2}+\vec{q}^{2}\right)$ at $|\vec{q}| \simeq 400 \mathrm{MeV} / c$, the most relevant momentum transfer in the weak decay transition. By construction, this type of monopole FF is equivalent to the exponential one at $\vec{q}=\overrightarrow{0}$ and we have verified that, up to a momentum transfer of $600 \mathrm{MeV} / c$ above which the transition amplitude is negligible, the differences between both functions are less than $2 \%$. The modified cutoffs, $\tilde{\Lambda}_{i}$, to be used in the monopole-type FF are listed in Table III. We point out

TABLE III. Cutoff values (in MeV) used in the present calculation for a FF of the type $\tilde{\Lambda}_{i}^{2} /\left(\widetilde{\Lambda}_{i}^{2}+\vec{q}^{2}\right)$, which matches the exponential-type FF used in Ref. [13].

\begin{tabular}{cccccc}
\hline \hline$\pi$ & $\eta$ & $K$ & $\rho$ & $\omega$ & $K^{*}$ \\
\hline 1750 & 1750 & 1789 & 1232 & 1310 & 1649 \\
\hline \hline
\end{tabular}

that the cutoff for the pion form factor may appear rather large; however, Ref. [19] discusses how, within the framework of a particular model, the $\pi N N$ cutoff can be as large as $2 \mathrm{GeV}$ if the meson-exchange contributions included are not correlated.

To incorporate the effects of the strong baryon-baryon $(B B)$ interaction we solve a $T$-matrix scattering equation in momentum space for the outgoing two-particle system $(N N$ or $Y N)$. For the initial two-particle system $(\Lambda N$ or $\Lambda \Lambda)$ we take into account medium effects, thus intermediate states only propagate into states allowed by the Pauli operator ( $G$ matrix). For the initial interacting $\Lambda N$ pair we previously found [20] that multiplying the independent two-particle wave function by a spin-independent correlation function of the type

$$
f(r)=\left(1-e^{-r^{2} / a^{2}}\right)^{n}+b r^{2} e^{-r^{2} / c^{2}},
$$

with $a=0.5 \mathrm{fm}, b=0.25 \mathrm{fm}^{-2}, c=1.28 \mathrm{fm}, n=2$, produced a correlated wave function which averaged the ones obtained from a microscopic finite-nucleus $G$-matrix calculation [21] using the soft-core and hard-core Nijmegen models [22]. In the case of the interacting $\Lambda \Lambda$ system, we again assume a correlation function of the type of Eq. (12). The parameters, $a=0.80 \mathrm{fm}, b=0.12 \mathrm{fm}^{-2}, c=1.28 \mathrm{fm}$, and $n=1$, are determined to reproduce the ratio between the correlated and uncorrelated ${ }^{1} S_{0} \Lambda \Lambda$ wave functions in nuclear matter at saturation density, $\Psi_{{ }^{1} S_{0}}(k, r) / j_{0}(k r)$, taking $k$ $=100 \mathrm{MeV} / c$ as a representative momentum of the two $\Lambda$ 's in a finite nucleus. The nuclear-matter correlated wave function, $\Psi_{{ }^{1} S_{0}}(k, r)$, has been obtained from a coupled-channel $G$-matrix calculation using the NSC97f model of the new Nijmegen potentials. In Fig. 2, we show the probability per unit length of finding two $\Lambda$ particles at a distance $r$, given by $4 \pi r^{2}\left|\Psi_{\Lambda \Lambda}^{\text {rel }}\right|^{2}$. The solid line corresponds to the uncorrelated two- $\Lambda$ wave function, which in this work is given by the $1 s$ harmonic oscillator wave function with the parameter $b_{\text {rel }}=\sqrt{2} b_{\Lambda}$. The dashed line incorporates the calculated correlation function, $\Psi_{{ }^{1} S_{0}}(k, r) / j_{0}(k r)$, a result that is well parametrized by a phenomenological correlation function of the form of Eq. (12), as shown by the dotted line. 


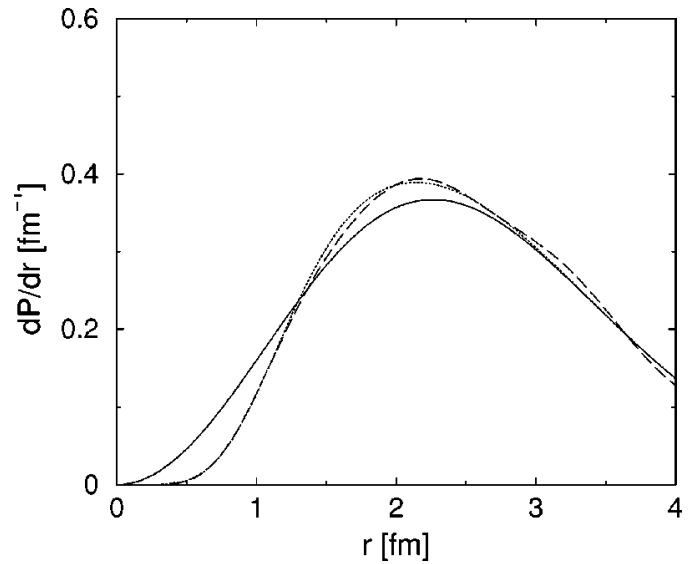

FIG. 2. Probability per unit length of finding two $\Lambda$ 's at a relative distance $r$.

As mentioned before, the strong BBM couplings are taken from the NSC97f [13] potential. In the weak sector only the couplings corresponding to decays involving pions are experimentally known. The weak couplings for heavy mesons are obtained following Refs. [2,23]. The PV amplitudes for the nonleptonic decays $B \rightarrow B^{\prime}+M$ involving pseudoscalar mesons are derived using the soft-meson reduction theorem and SU(3) symmetry. This allows us to relate the physical amplitudes of the nonleptonic hyperon decays into a pion plus a nucleon, $B \rightarrow B^{\prime}+\pi$, with the unphysical amplitudes involving other members of the meson and baryon octets. On the other hand, $\mathrm{SU}(6)_{W}$ permits relating the amplitudes involving pseudoscalar mesons with those for vector mesons. The weak PC coupling constants are obtained using a pole model, where the pole terms due to the $\left(\frac{1}{2}\right)^{+}$ground state (singular in the $\mathrm{SU}(3)$ soft meson limit) are assumed to be the dominant contribution. No meson pole terms are included. The values of these couplings for the weak vertices are listed in Table IV.

\section{RESULTS}

The results for the decay rates of ${ }_{\Lambda \Lambda}^{6} \mathrm{He}$ into the different channels are summarized in Tables V, VI, and VII, where the rate is presented in units of the free $\Lambda$ decay, $\Gamma_{\Lambda}=3.8$ $\times 10^{8} \mathrm{~s}^{-1}$. Apart from giving the various partial rates, the ratio between the neutron-induced and proton-induced decay rates, $\Gamma_{n} / \Gamma_{p}$, is also quoted. Only $\Delta I=1 / 2$ transitions have been considered.

We begin by discussing the results of Table $\mathrm{V}$, which list calculations absent of either strong correlations (initial and final) and FF's. Although the results are clearly unrealistic, they serve to illustrate, in comparison with Table VI, the effect of the strong interaction on the weak decay process. The $\Lambda N \rightarrow N N$ transitions receive contributions mainly from the $\pi, \rho, K$, and $K^{*}$ mesons; however, once short-range strong correlations are included (see Table VI), the role of the heavier mesons is strongly reduced. The results in Table $\mathrm{V}$ illustrate that isospin symmetry forbids isovector meson contributions, $(\pi, \rho)$, for the uncorrelated $\Lambda \Lambda \rightarrow \Lambda n$ decay rate and isoscalar meson contributions, $(\eta, \omega)$, for the uncor-
TABLE IV. Parity conserving (PC) and parity violating (PV) weak coupling constants for the decay of ${ }_{\Lambda \Lambda}^{6} \mathrm{He}$. The numbers are in units of $G_{F} m_{\pi}^{2}=2.21 \times 10^{-7}$. The NSC97f [13] model has been used for the strong sector.

\begin{tabular}{ccc}
\hline \hline & PV $(S$ wave $)$ & PC $(P$ wave $)$ \\
\hline$\Lambda n \pi^{0}$ & -1.05 & 7.15 \\
$\Lambda p \pi^{-}$ & 1.48 & -10.11 \\
$\Lambda n \eta$ & 1.80 & -11.90 \\
$p n K^{+}$ & 0.76 & -23.70 \\
$p p K^{0}$ & 2.09 & 8.33 \\
$n n K^{0}$ & 2.85 & -15.37 \\
$\Lambda \Lambda K^{0}$ & 0.67 & 12.72 \\
$\Lambda \Sigma^{0} K^{0}$ & 0.39 & 5.95 \\
$\Lambda \Sigma^{-} K^{+}$ & -0.55 & -8.42 \\
$\Lambda n \rho^{0}$ & -1.09 & $(\mathrm{~V}) 3.29$ \\
& & $(\mathrm{~T}) 6.74$ \\
$\Lambda p \rho^{-}$ & 1.54 & $(\mathrm{~V})-4.65$ \\
& & $(\mathrm{~T})-9.53$ \\
$\Lambda n \omega$ & -1.33 & $(\mathrm{~V})-0.17$ \\
& & $(\mathrm{~T})-7.43$ \\
$p p K^{* 0}$ & 0.60 & $(\mathrm{~V})-5.46$ \\
& & $(\mathrm{~T}) 6.23$ \\
$p n K^{*+}$ & -4.48 & $(\mathrm{~V})-4.02$ \\
$n n K^{* 0}$ & -3.88 & $(\mathrm{~T})-19.54$ \\
& & $(\mathrm{~V})-9.48$ \\
$\Lambda \Lambda K^{* 0}$ & -1.38 & $(\mathrm{~T})-13.31$ \\
$\Lambda \Sigma^{0} K^{* 0}$ & & $(\mathrm{~V})-1.34$ \\
$\Lambda \Sigma^{-} K^{*+}$ & 0.63 & $(\mathrm{~T}) 11.20$ \\
& -0.88 & $(\mathrm{~V})-3.90$ \\
\hline \hline
\end{tabular}

related $\Lambda \Lambda \rightarrow \Sigma N$ rates. Finally, we note that the nucleoninduced rate is about 20 times larger than the total hyperoninduced contribution.

When FF's and strong correlations in the initial and final states are included, the picture changes considerably, as can be seen from the results shown in Table VI. Most of the $\Lambda N \rightarrow N N$ rate comes from the exchange of the $\pi$ and $K$ mesons. Note that the small $\Gamma_{n} / \Gamma_{p}$ ratio for the one-pion exchange mechanism substantially increases when the $K$-meson is incorporated due to a destructive (constructive) interference in the $p$-induced ( $n$-induced) rate. This aspect is discussed recently in Ref. [4], which updates the mesonexchange model of Ref. [3] and finds agreements with results obtained by groups combining the $\pi$ and $K$ exchange with either a direct quark mechanism [5] or $2 \pi$ exchange [24]. Since there are now two $\Lambda$ particles contributing to the $\Lambda N$ $\rightarrow N N$ decay, one would expect the result to be roughly twice the rate in ${ }_{\Lambda}^{5} \mathrm{He}$, which for this potential turns out to be $0.32 \Gamma_{\Lambda}$, as shown in Ref. [4]. We obtain instead a significantly larger value of $\Gamma_{N N}=0.96 \Gamma_{\Lambda}$. This finding can be traced to the $\Lambda$ that is more strongly bound in ${ }_{\Lambda \Lambda}^{6} \mathrm{He}$ than it is in ${ }_{\Lambda}^{5} \mathrm{He}$ by about $80 \%$ (i.e., the $\Lambda$ single-particle separation energy changes from $3.12 \mathrm{MeV}$ to around $5 \mathrm{MeV}$ ). This 
TABLE V. Individual and combined meson-exchange contributions to the nonmesonic weak decay of ${ }_{\Lambda \Lambda}^{6} \mathrm{He}$ in absence of either strong correlations and FF. The nonmesonic decay rate is in units of $\Gamma_{\Lambda}=3.8$ $\times 10^{9} \mathrm{~s}^{-1}$. The NSC97f [13] strong coupling constants have been used.

\begin{tabular}{|c|c|c|c|c|c|c|c|}
\hline \multirow[b]{2}{*}{ Meson } & \multicolumn{3}{|c|}{$\Gamma_{N N}$} & \multirow[t]{2}{*}{$\Gamma_{\Lambda n}$} & \multicolumn{2}{|c|}{$\Gamma_{\Sigma N}$} & \multirow[t]{2}{*}{$\Gamma_{N N}+\Gamma_{Y N}$} \\
\hline & $\Gamma_{n n}$ & $\Gamma_{n p}$ & $\Gamma_{n} / \Gamma_{p}$ & & $\Gamma_{\sum 0_{n}}$ & $\Gamma_{\Sigma^{-} p}$ & \\
\hline$\pi$ & 0.40 & 2.08 & 0.19 & -- & $2.1 \times 10^{-2}$ & $4.2 \times 10^{-2}$ & 2.54 \\
\hline K & 0.67 & 1.14 & 0.58 & $3.5 \times 10^{-2}$ & $2.3 \times 10^{-3}$ & $4.6 \times 10^{-3}$ & 1.85 \\
\hline$\eta$ & $3.5 \times 10^{-2}$ & $3.0 \times 10^{-2}$ & 1.18 & $2.0 \times 10^{-3}$ & -- & -- & $6.6 \times 10^{-2}$ \\
\hline$\rho$ & 0.30 & 0.70 & 0.42 & -- & $2.2 \times 10^{-3}$ & $4.5 \times 10^{-3}$ & 1.00 \\
\hline$K^{*}$ & 3.46 & 2.31 & 1.50 & $1.6 \times 10^{-2}$ & $3.8 \times 10^{-2}$ & $7.7 \times 10^{-2}$ & 5.90 \\
\hline$\omega$ & $8.6 \times 10^{-2}$ & $6.6 \times 10^{-2}$ & 1.31 & $6.8 \times 10^{-3}$ & -- & -- & 0.16 \\
\hline$\pi+K$ & 0.96 & 1.09 & 0.88 & $3.5 \times 10^{-2}$ & $1.8 \times 10^{-2}$ & $3.5 \times 10^{-2}$ & 2.13 \\
\hline$\pi+K+\eta$ & 1.19 & 1.06 & 1.12 & $2.6 \times 10^{-2}$ & $1.8 \times 10^{-2}$ & $3.5 \times 10^{-2}$ & 2.34 \\
\hline all & 2.45 & 4.29 & 0.57 & 0.10 & $9.0 \times 10^{-2}$ & 0.18 & 7.12 \\
\hline
\end{tabular}

increased binding is reflected in the $\Lambda$ single-particle wave function, with a harmonic oscillator parameter $b_{\Lambda}=1.6 \mathrm{fm}$ instead of the value $b_{\Lambda}=1.87 \mathrm{fm}$ used in the decay of single- $\Lambda$ hypernuclei $[3,4]$. As mentioned in Sec. II, the parameter $b_{\Lambda}=1.6 \mathrm{fm}$ simulates the uncorrelated $\Lambda \Lambda$ probability of Ref. [17], which reproduces the binding energy of the three observed double- $\Lambda$ hypernuclei. There is also some influence from the slightly different kinematics involved in the $\Lambda N \rightarrow N N$ decay of ${ }_{\Lambda \Lambda}^{6} \mathrm{He}$ with respect to that in ${ }_{\Lambda}^{5} \mathrm{He}$.

From the results presented in Table VI, we observe that the $\Lambda \Lambda \rightarrow \Lambda n$ rate receives a tiny contribution from the isovector $\pi$ and $\rho$ mesons. This is due to the combined transition $\Lambda \Lambda \stackrel{\text { weak }}{\rightarrow} \Sigma N \rightarrow \Lambda$ strong $\rightarrow$, which is possible through the coupling between $\Lambda N$ and $\Sigma N$ states induced by the strong interaction. Analogously, the strong $\Lambda N-\sum N$ coupling also induces very small contributions from the isoscalar mesons to the $\Sigma N$ rate, $\Gamma_{\Sigma N}$.
We find the rate $\Gamma_{\Lambda n}$ to be dominated by $K$ exchange. We note that the $\pi+K$ contribution is very similar to the value for $K$ exchange alone; adding the $\eta$ meson decreases the rate by $35 \%$. However, there is strong evidence that we are overestimating the role of the $\eta$ meson by using strong $\eta N N$ and $\eta \Lambda \Lambda$ couplings that rely on $\mathrm{SU}(3)$ symmetry. All indications from a careful analysis of reactions like $\eta$ photoproduction [25] point towards a $\eta N N$ coupling much smaller than the one provided by $\mathrm{SU}(3)$ symmetry. In this case, the weak decay of double- $\Lambda$ hypernuclei going to final $\Lambda n$ states would be almost solely determined by $K$ exchange and the measurement of this decay channel would provide valuable information on the weak $\Lambda \Lambda K$ couplings. In particular, since this partial rate is dominated by PC transitions, as can be inferred from the values of the $\Lambda \Lambda K^{0}$ weak couplings listed in Table IV, one would gain access to the PC $\Lambda \Lambda K^{0}$ amplitude. The rate to final $\Sigma N$ states, $\Gamma_{\Sigma N}$, on the other hand, is

TABLE VI. Individual and combined meson-exchange contributions to the nonmesonic weak decay of ${ }_{\Lambda \Lambda}^{6} \mathrm{He}$. Strong correlations (initial and final) and FF are included. The nonmesonic decay rate is in units of $\Gamma_{\Lambda}=3.8 \times 10^{9} \mathrm{~s}^{-1}$. The NSC97f [13] strong interaction model has been used. The values between parentheses have been obtained using the weak kaon couplings from Refs. [31,32].

\begin{tabular}{|c|c|c|c|c|c|c|c|}
\hline \multirow[b]{2}{*}{ Meson } & \multicolumn{3}{|c|}{$\Gamma_{N N}$} & \multirow[t]{2}{*}{$\Gamma_{\Lambda n}$} & \multicolumn{2}{|c|}{$\Gamma_{\Sigma N}$} & \multirow[t]{2}{*}{$\Gamma_{N N}+\Gamma_{Y N}$} \\
\hline & $\Gamma_{n n}$ & $\Gamma_{n p}$ & $\Gamma_{n} / \Gamma_{p}$ & & $\Gamma_{\Sigma^{0} n}$ & $\Gamma_{\Sigma^{-} p}$ & \\
\hline$\pi$ & $9.9 \times 10^{-2}$ & 1.09 & $9.1 \times 10^{-2}$ & $1.3 \times 10^{-4}$ & $1.8 \times 10^{-3}$ & $3.7 \times 10^{-3}$ & 1.19 \\
\hline \multirow[t]{2}{*}{$K$} & $8.2 \times 10^{-2}$ & 0.47 & 0.17 & $2.7 \times 10^{-2}$ & $3.1 \times 10^{-4}$ & $6.1 \times 10^{-4}$ & 0.58 \\
\hline & $\left(5.3 \times 10^{-2}\right)$ & $(0.11)$ & $(0.50)$ & $\left(1.3 \times 10^{-2}\right)$ & $\left(1.7 \times 10^{-5}\right)$ & $\left(3.4 \times 10^{-5}\right)$ & $(0.17)$ \\
\hline$\eta$ & $3.9 \times 10^{-3}$ & $7.6 \times 10^{-3}$ & 0.51 & $1.1 \times 10^{-3}$ & $2.5 \times 10^{-7}$ & $5.1 \times 10^{-7}$ & $1.3 \times 10^{-2}$ \\
\hline$\rho$ & $7.4 \times 10^{-3}$ & $2.5 \times 10^{-2}$ & 0.30 & $4.7 \times 10^{-6}$ & $2.0 \times 10^{-6}$ & $4.0 \times 10^{-6}$ & $3.2 \times 10^{-2}$ \\
\hline$K^{*}$ & $3.8 \times 10^{-3}$ & $2.5 \times 10^{-2}$ & 0.15 & $4.8 \times 10^{-3}$ & $7.3 \times 10^{-5}$ & $1.5 \times 10^{-4}$ & $3.4 \times 10^{-2}$ \\
\hline$\omega$ & $1.1 \times 10^{-3}$ & $0.95 \times 10^{-3}$ & 0.12 & $7.2 \times 10^{-5}$ & $2.5 \times 10^{-8}$ & $5.0 \times 10^{-8}$ & $2.1 \times 10^{-3}$ \\
\hline \multirow[t]{2}{*}{$\pi+K$} & 0.24 & 0.48 & 0.50 & $2.6 \times 10^{-2}$ & $1.4 \times 10^{-3}$ & $2.9 \times 10^{-3}$ & 0.75 \\
\hline & $(0.21)$ & $(0.82)$ & $(0.25)$ & $\left(1.3 \times 10^{-2}\right)$ & $\left(2.2 \times 10^{-3}\right)$ & $\left(4.4 \times 10^{-3}\right)$ & $(1.05)$ \\
\hline \multirow[t]{2}{*}{$\pi+K+\eta$} & 0.27 & 0.44 & 0.61 & $1.7 \times 10^{-2}$ & $1.4 \times 10^{-3}$ & $2.8 \times 10^{-3}$ & 0.73 \\
\hline & $(0.24)$ & $(0.73)$ & $(0.33)$ & $\left(6.6 \times 10^{-3}\right)$ & $\left(2.1 \times 10^{-3}\right)$ & $\left(4.3 \times 10^{-3}\right)$ & $(0.99)$ \\
\hline \multirow[t]{2}{*}{ All } & 0.30 & 0.66 & 0.46 & $3.6 \times 10^{-2}$ & $1.3 \times 10^{-3}$ & $2.6 \times 10^{-3}$ & 1.00 \\
\hline & $(0.25)$ & (1.13) & $(0.23)$ & $\left(1.9 \times 10^{-2}\right)$ & $\left(1.7 \times 10^{-3}\right)$ & $\left(3.4 \times 10^{-3}\right)$ & (1.41) \\
\hline
\end{tabular}


TABLE VII. Decay rate of ${ }_{\Lambda \Lambda}^{6} \mathrm{He}$ in units of $\Gamma_{\Lambda}=3.8 \times 10^{9} \mathrm{~s}^{-1}$ for different approaches to FSI. All mesons are included in the calculation.

\begin{tabular}{|c|c|c|c|c|c|c|c|}
\hline & \multicolumn{3}{|c|}{$\Gamma_{N N}$} & \multirow[t]{2}{*}{$\Gamma_{\Lambda n}$} & \multicolumn{2}{|c|}{$\Gamma_{\Sigma N}$} & \multirow[t]{2}{*}{$\Gamma_{N N}+\Gamma_{Y N}$} \\
\hline & $\Gamma_{n n}$ & $\Gamma_{n p}$ & $\Gamma_{n} / \Gamma_{p}$ & & $\Gamma_{\Sigma^{0} n}$ & $\Gamma_{\Sigma^{-} p}$ & \\
\hline NO FSI & 0.69 & 1.21 & 0.57 & $2.5 \times 10^{-3}$ & $1.4 \times 10^{-2}$ & $2.8 \times 10^{-2}$ & 1.94 \\
\hline FSI eff. & 0.73 & 1.27 & 0.58 & $4.4 \times 10^{-3}$ & $1.1 \times 10^{-2}$ & $2.2 \times 10^{-2}$ & 2.04 \\
\hline$T$ matrix & 0.30 & 0.66 & 0.46 & $3.6 \times 10^{-2}$ & $1.3 \times 10^{-3}$ & $2.6 \times 10^{-3}$ & 1.00 \\
\hline
\end{tabular}

very small and, as expected, dominated by the $\pi$-exchange mechanism.

The ratios between the rates that include correlations are

$$
\frac{\Gamma_{N N}}{\Gamma_{\Sigma N}}: \frac{\Gamma_{\Lambda N}}{\Gamma_{\Sigma N}}: \frac{\Gamma_{\Sigma N}}{\Gamma_{\Sigma N}} \simeq 246: 9: 1
$$

Correlations have a stronger influence on the $\mathrm{Y} N$ channels, which are a factor 30-300 smaller compared to the $N N$ rate, even though the $\Sigma N$ transition allows a final state with a lower relative momentum and therefore is less sensitive to the strongly repulsive core of the $Y N$ interaction. However, the $N N$ rate is dominated by tensor transitions that are absent in the $Y N$ channels because the interacting $\Lambda \Lambda$ pair is in a ${ }^{1} S_{0}$ state. With the tensor strength distributed towards larger distances compared to those for central and parity-violating transitions, the $N N$ transition becomes comparatively less affected by strong correlations than the $Y N$ ones. Note that correlations tend to increase the $\Lambda N$ rate while decreasing the $\Sigma N$ rate. The reason is that the uncorrelated $\Sigma N$ rate is dominated by $\pi$ and $K^{*}$ exchanges, the $K^{*}$ contribution being about twice that of the $\pi$ meson, whereas the $\Lambda N$ rate comes mainly from strange meson exchange, with the $K$ contribution twice as large as that of the $K^{*}$. Therefore, the supression of short-range physics induced by correlations affects the $\Sigma N$ rate more than the $\Lambda N$ one. In addition, it is known $[26,27]$ that the $\Sigma N$ interaction for the new Nijmegen potentials is quite repulsive in the $I=1 / 2$ channel, affecting the $\Sigma N$ wave functions significantly, which are pushed out from the weak potential interaction range.

The results for various approaches to the treatment of FSI are compared in Table VII. Those labeled with "FSI eff." have been obtained by multiplying the free $N N, \Lambda N$, and $\Sigma N$ final states by the momentum and channel independent function $1-j_{0}\left(q_{c} r\right)$, with $q_{c}=3.93 \mathrm{fm}^{-1}$. As recently discussed in Ref. [4], treating FSI in this simplified phenomenological fashion overestimates the rate $\Gamma_{N N}$ calculated rigorously with a $T$ matrix by about a factor of 2 . The rate $\Gamma_{\Lambda \mathrm{n}}$ obtained with the phenomenological FSI treatment is almost one order of magnitude smaller compared with the result obtained within a $T$-matrix approach, while on the other hand, the rate $\Gamma_{\Sigma N}$ is overpredicted by almost an order of magnitude. In the same reference, one compares the effects of using different versions of the NSC97 potentials in the decay observables. One of the results of that work is that working consistently within the NSC97a or the NSC97f potential models, the decay rate changes by $\approx 25 \%$ (study made for two hypernuclei ${ }_{\Lambda}^{5} \mathrm{He}$ and ${ }_{\Lambda}^{12} \mathrm{C}$ ), the neutron-to- proton ratio by $26 \%$ for ${ }_{\Lambda}^{5} \mathrm{He}$ and $15 \%$ for ${ }_{\Lambda}^{12} \mathrm{C}$, while the PV asymmetry remains practically unchanged. As in Ref. [4], we again find that in order to be able to extract information on the weak decay amplitudes a careful treatment of FSI is essential in the study of the weak decay of double- $\Lambda$ hypernuclei. On the other hand, we have also studied the sensitivity of our results to the $\pi N N$ cutoff, listed in Table III. Lowering the cutoff from $1700 \mathrm{MeV}$ down to $1300 \mathrm{MeV}$ we find that, once the strong initial- and final-state interactions are included, our results change by less than $10 \%$.

We finally compare our results with the ones presented recently by Itonaga et al. [28], where the weak decay mechanism contains $\pi$ and $\rho$ and correlated two-pion exchange with scalar $(\sigma)$ and vector $(\rho)$ quantum numbers. The $\Lambda$ wave function in ${ }_{\Lambda \Lambda}^{6} \mathrm{He}$ was taken the same as in their ${ }_{\Lambda}^{5} \mathrm{He}$ calculation [29], thus neglecting the possible effect of a stronger binding of the $\Lambda$ in a double- $\Lambda$ hypernucleus. Hence, the nucleon-induced rate obtained in Ref. [28] is $\Gamma_{N N}=0.753 \Gamma_{\Lambda}$, equal to twice their decay rate in ${ }_{\Lambda}^{5} \mathrm{He}$. This rate is about $20 \%$ smaller than the one obtained here. The rates for $\Lambda n$ and $\Sigma N$ final states, $\Gamma_{\Lambda n}=0.025 \Gamma_{\Lambda}$ and $\Gamma_{\Sigma N}$ $=0.0012 \Gamma_{\Lambda}$, are about a factor of 2 smaller than the ones obtained in this work. We note that the $\Lambda \Lambda$ correlation function used in Ref. [28] is in fact the ${ }^{1} S_{0} \Lambda N$ one used in their study of the decay of ${ }_{\Lambda}^{5} \mathrm{He}$. We also note that the $K$-exchange mechanism, dominant in the $\Lambda \Lambda \rightarrow \Lambda n$ transition amplitude, is absent in the work of Ref. [28].

In Ref. [3], we explored SU(3) symmetry breaking effects in the decay of single- $\Lambda$ hypernuclei by using weak $N N K$ couplings derived in the framework of heavy baryon chiral perturbation theory [30], where one-loop SU(3) corrections to leading order were evaluated. Here we explore similar effects not only for the $N N K$ but also for the $\Lambda \Lambda K$ and $\Lambda \Sigma K$ couplings, which were calculated in Refs. [31,32]. In Table VIII we list the values for the weak PV ( $S$-wave) and

TABLE VIII. $\chi \mathrm{PT}$ one-loop corrected $N N K, \Lambda \Lambda K$, and $\Lambda \Sigma K$ couplings. Values taken from Refs. [31,32].

\begin{tabular}{lcc}
\hline \hline & $S$ wave & $P$ wave \\
\hline$p n K^{+}$ & $(0.32 \pm 0.24)$ & $(-10.41 \pm 1.61)$ \\
$p p K^{0}$ & $(2.32 \pm 0.22)$ & $(3.47 \pm 2.23)$ \\
$n n K^{0}$ & $(2.64 \pm 0.25)$ & $(-6.94 \pm 2.23)$ \\
$\Lambda \Lambda K^{0}$ & $(0.78 \pm 0.20)$ & $(8.69 \pm 2.21)$ \\
$\Lambda \Sigma^{0} K^{0}$ & $(0.53 \pm 0.12)$ & $(-0.58 \pm 1.83)$ \\
$\Lambda \Sigma^{-} K^{+}$ & $(-0.75 \pm 0.17)$ & $(0.82 \pm 2.59)$ \\
\hline
\end{tabular}


TABLE IX. Partial weak decay rates for ${ }_{\Lambda \Lambda}^{6} \mathrm{He}$ (in units of $\Gamma_{\Lambda}$ $=3.8 \times 10^{9} \mathrm{~s}^{-1}$ ).

\begin{tabular}{lcc}
\hline \hline & NSC97f & NSC97f + one-loop corrections \\
\hline$\Lambda n \rightarrow n n$ & 0.30 & $0.25 \pm 15 \%$ \\
$\Lambda p \rightarrow n p$ & 0.66 & $1.13 \pm 15 \%$ \\
$\Lambda N \rightarrow N N$ & 0.96 & $1.38 \pm 15 \%$ \\
$\Gamma_{n} / \Gamma_{p}$ & 0.46 & $0.23 \pm 20 \%$ \\
$\Lambda \Lambda \rightarrow \Lambda n$ & $3.6 \times 10^{-2}$ & $1.9 \times 10^{-2} \pm 50 \%$ \\
$\Lambda \Lambda \rightarrow \Sigma^{0} n$ & $1.3 \times 10^{-3}$ & $1.7 \times 10^{-3} \pm 20 \%$ \\
$\Lambda \Lambda \rightarrow \Sigma^{-} p$ & $2.6 \times 10^{-3}$ & $3.4 \times 10^{-3} \pm 20 \%$ \\
$\Lambda \Lambda \rightarrow Y N$ & $4.0 \times 10^{-2}$ & $2.4 \times 10^{-2} \pm 20 \%$ \\
\hline \hline
\end{tabular}

PC ( $P$-wave) couplings involving the kaon. Compared to the ones quoted in Ref. [3], the numbers in Table VIII differ since they were obtained using a common mass splitting of $200 \mathrm{MeV}$ between the octet and decuplet baryons [31,32].

Comparing the values listed in Table VIII with those in Table IV we observe that the one-loop corrected $N N K$ $P$-wave constants are approximately a factor of 2 smaller than the tree level ones, while the $S$-wave are very similar, except for the $p n K^{+}$coupling, which, again, is half the treelevel value. This explains the reduced $K$-exchange contribution to the nucleon-induced rates with respect to the treelevel result. Note that the magnitude of the (constructive) interference between $\pi$ and $K$ in the $n n$ channel has not changed much, while it has become less destructive in the $n p$ channel, thereby producing a smaller $\Gamma_{\mathrm{n}} / \Gamma_{\mathrm{p}}$ ratio. Once all the mesons are included, the $N N$ rate obtained with the oneloop corrected $N N K$ couplings is a factor of 1.4 larger than that obtained with the tree-level values, while the ratio $\Gamma_{n} / \Gamma_{p}$ is smaller by a factor of 2 .

Although the one-loop corrected $\Lambda \Lambda K$ couplings of Table VIII are not much different from the corresponding tree-level values, they lead to a reduction of the $K$-meson exchange contribution to the $\Lambda n$ rate by about a factor of 2 . Hence, the contribution of the $\eta$ meson and the vector mesons becomes more relevant, making the extraction of the $\Lambda \Lambda \mathrm{K}$ coupling from this $\Lambda \Lambda \rightarrow \Lambda n$ decay mode more difficult.

The decay rate into final $\Sigma N$ states is the only one that increases when the one-loop corrected $\Lambda \Sigma K$ couplings are used, despite the magnitude of the $P$-wave amplitudes being ten times smaller than the corresponding tree-level values, whereas the $S$-wave amplitudes are only moderately larger. In fact, Table VI reveals that the $K$-exchange contribution to the $\Sigma N$ rate is two times smaller than that obtained with the tree-level $\Lambda \Sigma K$ couplings, as expected from the reduced magnitude of the couplings. However, due to the opposite sign of the loop-corrected $P$-wave coupling with respect to the corresponding tree-level value, the interference between the $\pi$ and $K$ contributions is now constructive instead of destructive, which explains the final increased loop-corrected rate.

Finally, we have investigated how our results would change if we allowed the one-loop corrected $B B K$ couplings to vary within their estimated error bands. The corresponding range of variation on the rates is quoted in Table IX, where the results obtained with the tree-level $B B K$ couplings have also been included to facilitate the comparison between both calculations. The results summarized in Table IX show clearly that the inclusion of weak BBK couplings obtained with one-loop corrections to the leading order in $\chi \mathrm{PT}$ produces a $\Lambda N \rightarrow N N$ rate $40 \%$ larger than the tree-level value, a ratio $\Gamma_{n} / \Gamma_{p}$ twice smaller, a $\Lambda \Lambda \rightarrow \Lambda n$ rate roughly twice smaller, and a $\Lambda \Lambda \rightarrow \Sigma N$ rate $30 \%$ larger. We acknowledge that, since at the present time $\chi \mathrm{PT}$ has little predictive power for weak meson-baryon-baryon couplings, these comparisons serve to demonstrate that the nonmesonic weak decay mode is indeed suitable for the extraction of these weak vertices.

\section{CONCLUSIONS}

In this study we investigated the nonmesonic weak decay modes of double- $\Lambda$ hypernuclei within a one-mesonexchange framework. Our results are given for the specific double- $\Lambda$ hypernucleus ${ }_{\Lambda \Lambda}^{6} \mathrm{He}$, but can easily be extended to heavier systems.

The standard nucleon-induced nonmesonic $\Lambda N \rightarrow N N$ rate is found to be more than twice as large as in ${ }_{\Lambda}^{5} \mathrm{He}$ due to the increased binding of the second $\Lambda$ hyperon. Two new hyperon-induced modes become possible, $\Lambda \Lambda \rightarrow \Lambda n$ and $\Lambda \Lambda \rightarrow \Sigma N$, the latter coming in two charge states. We find the total hyperon-induced rate to be as large as $4 \%$ of the total nonmesonic rate. This new rate is dominated by the $\Lambda \Lambda \rightarrow \Lambda n$ mode. In fact, this transition turns out to be the more interesting one (rather than the $\Lambda \Lambda \rightarrow \Sigma N$ ) since it allows direct access to exotic vertices like $\Lambda \Lambda K$, unencumbered by the usually dominant pion exchange. Indeed, oneloop log-corrected $\chi$ PT results modify the $\Lambda \Lambda \rightarrow \Lambda n$ by $50 \%$ while changing the $\Lambda N \rightarrow N N$ only at the $15 \%$ level, demonstrating the power of this weak mechanism to test $\chi \mathrm{PT}$ in the weak SU(3) sector. With a free $\Lambda$ in the final state this new mode should be distinguishable from the usual nucleoninduced decay channels. Given the potential benefits to hadronic physics, the experimental program of investigating the production and decay of double- $\Lambda$ hypernuclei should be intensified.

\section{ACKNOWLEDGMENTS}

The authors would like to thank Christoph Hanhart, Barry R. Holstein, Jürgen Schaffner-Bielich, Roxanne P. Springer, and Isaac Vidaña for their input and comments on different aspects of the present paper, regarding both the strong and weak baryon-baryon interaction. One of the authors, A.P., would like to thank the Institute for Nuclear Theory and the University of Washington (Seattle) where an important part of this work was developed. This work has been partially supported by the U.S. Department of Energy under Grant Nos. DE-FG03-00-ER41132 and DE-FG02-95ER-40907, by the DGICYT (Spain) under Contract No. PB98-1247, and by the Generalitat de Catalunya Project No. SGR2000-24. 


\section{APPENDIX}

\section{Weak PC couplings}

In this subsection we present the expression for the PC weak coupling constants derived by using a pole model [2] with only baryon poles.

For $\pi$ exchange,

$\mathcal{A}\left(\Lambda, n \pi^{0}\right)=g\left(n, n \pi^{0}\right) \frac{A_{\Lambda n}}{m_{\Lambda}-m_{n}}+g\left(\Lambda, \Sigma^{0} \pi^{0}\right) \frac{A_{\Sigma^{0} n}}{m_{n}-m_{\Sigma^{0}}}$,

$\mathcal{A}\left(\Lambda, p \pi^{-}\right)=g\left(n, p \pi^{-}\right) \frac{A_{\Lambda n}}{m_{\Lambda}-m_{n}}+g\left(\Lambda, \Sigma^{+} \pi^{-}\right) \frac{A_{\Sigma^{+} p}}{m_{p}-m_{\Sigma^{+}}}$.

For $\eta$ exchange,

$$
\mathcal{A}(\Lambda, n \eta)=g(n, n \eta) \frac{A_{\Lambda n}}{m_{\Lambda}-m_{n}}+g(\Lambda, \Lambda \eta) \frac{A_{\Lambda n}}{m_{n}-m_{\Lambda}} .
$$

For $K$ exchange,

$$
\begin{gathered}
\mathcal{A}\left(n, n K^{0}\right)=g\left(n, \Lambda K^{0}\right) \frac{A_{\Lambda n}}{m_{n}-m_{\Lambda}}+g\left(n, \Sigma^{0} K^{0}\right) \frac{A_{\Sigma^{0} n}}{m_{n}-m_{\Sigma^{0}}}, \\
\mathcal{A}\left(p, p K^{0}\right)=g\left(p, \Sigma^{+} K^{0}\right) \frac{A_{\Sigma^{+} p}}{m_{p}-m_{\Sigma^{+}}},
\end{gathered}
$$$$
\mathcal{A}\left(p, n K^{+}\right)=g\left(p, \Sigma^{0} K^{+}\right) \frac{A_{\Sigma^{0} n}}{m_{n}-m_{\Sigma^{0}}}+g\left(p, \Lambda K^{+}\right) \frac{A_{\Lambda n}}{m_{n}-m_{\Lambda}},
$$$$
\mathcal{A}\left(\Lambda, \Lambda K^{0}\right)=g\left(n, \Lambda K^{0}\right) \frac{A_{\Lambda n}}{m_{\Lambda}-m_{n}}+g\left(\Lambda, \Xi^{0} K^{0}\right) \frac{A_{\Xi^{0} \Lambda}}{m_{\Lambda}-m_{\Xi}^{0}},
$$

$$
\begin{gathered}
\mathcal{A}\left(\Lambda, \Sigma^{0} K^{0}\right)=g\left(n, \Sigma^{0} K^{0}\right) \frac{A_{\Lambda n}}{m_{\Lambda}-m_{n}}, \\
\mathcal{A}\left(\Lambda, \Sigma^{-} K^{+}\right)=g\left(n, \Sigma^{-} K^{+}\right) \frac{A_{\Lambda n}}{m_{\Lambda}-m_{n}} .
\end{gathered}
$$

To compute the former amplitudes, we use the following values for the weak transitions at the baryon lines $A_{B B^{\prime}}$ :

$$
\begin{gathered}
A_{\Lambda n} \equiv A_{\Lambda \mathrm{N}}=-4.32 \times 10^{-5} \mathrm{MeV}, \\
A_{\Sigma^{0} n} \equiv-A_{\Sigma \mathrm{N}}=4.35 \times 10^{-5} \mathrm{MeV}, \\
A_{\Xi^{0} \Lambda} \equiv A_{\Xi \Lambda}=5.93 \times 10^{-5} \mathrm{MeV}, \\
A_{\Sigma^{+} p} \equiv \sqrt{2} A_{\Sigma N}=-6.15 \times 10^{-5} \mathrm{MeV} .
\end{gathered}
$$

For the vector $\rho, \omega$, and $K^{*}$ mesons one has to make the following replacements in Eqs. (A1)-(A9):

$$
\begin{gathered}
\pi \rightarrow \rho, \\
\eta \rightarrow \omega, \\
K \rightarrow K^{*} .
\end{gathered}
$$

\section{Weak PV couplings}

SU(3) allows us to obtain the weak PV couplings involving two baryons and one pseudoscalar $(\pi, \eta, K)$ meson. In order to obtain the corresponding constants for vector mesons we use $\mathrm{SU}(6)_{W}$. Here, we quote the results for all the couplings that appear in the $\Lambda N \rightarrow N N$ and $\Lambda N \rightarrow Y N$ channels:

$$
\begin{gathered}
\left(\Lambda, p \pi^{-}\right)_{\exp } \equiv \Lambda_{-}^{0}=3.25 \times 10^{-7} \\
\left(\Sigma^{+}, p \pi^{0}\right)_{\exp } \equiv \Sigma_{0}^{+}=-3.27 \times 10^{-7}, \\
\left(\Lambda, n \pi^{0}\right)=-\frac{1}{\sqrt{2}} \Lambda_{-}^{0}
\end{gathered}
$$

$$
\left(\Lambda, n \rho^{0}\right)=\frac{1}{3}\left(-2 \Lambda_{-}^{0}+\sqrt{3} \Sigma_{0}^{+}-\sqrt{3} a_{T}\right),
$$

$$
\begin{gathered}
\left(\Lambda, p \rho^{-}\right)=\frac{1}{3} \sqrt{\frac{2}{3}}\left(2 \sqrt{3} \Lambda_{-}^{0}-3 \Sigma_{0}^{+}-3 a_{V}\right), \\
(\Lambda, n \eta)=\sqrt{\frac{3}{2}} \Lambda_{-}^{0},
\end{gathered}
$$

$$
(\Lambda, n \omega)=\Sigma_{0}^{+}-\frac{1}{3} a_{T}
$$

$$
\left(p, p K^{*^{0}}\right)=-\frac{2}{3} \Sigma_{0}^{+}+\frac{8}{9} a_{T},
$$

$$
\left(p, n K^{*^{+}}\right)=-\sqrt{3} \Lambda_{-}^{0}+\frac{1}{3} \Sigma_{0}^{+}+\frac{10}{9} a_{V},
$$

$$
\left(\Lambda, \Lambda K^{0}\right)=-\frac{1}{2} \sqrt{\frac{3}{2}} \Lambda_{-}^{0}-\frac{3}{2 \sqrt{2}} \Sigma_{0}^{+},
$$




$$
\begin{gathered}
\left(\Lambda, \Sigma^{0} K^{0}\right)=-\frac{1}{2 \sqrt{2}} \Lambda_{-}^{0}-\frac{1}{2} \sqrt{\frac{3}{2} \Sigma_{0}^{+},} \\
\left(\Lambda, \Sigma^{-} K^{+}\right)=\frac{1}{2} \Lambda_{-}^{0}+\frac{\sqrt{3}}{2} \Sigma_{0}^{+}, \\
\left(\Lambda, \Lambda K^{*^{0}}\right)=-\frac{5 \sqrt{3}}{6} \Lambda_{-}^{0}-\frac{1}{2} \Sigma_{0}^{+}, \\
\left(\Lambda, \Sigma^{0} K^{*}\right)=\frac{7}{6} \Lambda_{-}^{0}+\frac{3}{2 \sqrt{3}} \Sigma_{0}^{+}+\frac{2}{3 \sqrt{3}} a_{T}, \\
\left(\Lambda, \Sigma^{-} K^{*}\right)=-\frac{7}{3 \sqrt{2}} \Lambda_{-}^{0}-\frac{3}{\sqrt{6}} \Sigma_{0}^{+}+\frac{4}{3 \sqrt{6}} a_{V} .
\end{gathered}
$$

If we desire only the $\Delta I=\frac{1}{2}$ part of the above expressions, as we do in the present work, the following replacements have to be made [33]: For the $\Lambda N \rho$ couplings, $a_{V} \rightarrow 3 a_{V}$ and $a_{T} \rightarrow 3 a_{T}$. For the $\Lambda \Sigma \mathrm{K}^{*}$ couplings, $a_{V} \rightarrow\left(a_{V}-a_{T}\right) / \sqrt{3}$ and $a_{T} \rightarrow-\left(a_{V}-a_{T}\right) / \sqrt{3}$.

\section{Strong coupling constants}

In this subsection we present the convention used for the strong couplings.

$$
\begin{aligned}
g_{N N \pi} & \equiv g\left(p, p \pi^{0}\right)=-g\left(n, n \pi^{0}\right)=\frac{1}{\sqrt{2}} g\left(p, n \pi^{+}\right) \\
& =\frac{1}{\sqrt{2}} g\left(n, p \pi^{-}\right)
\end{aligned}
$$

$$
\begin{aligned}
g_{\Lambda \Sigma \pi} & \equiv g\left(\Sigma^{+}, \Lambda \pi^{+}\right)=g\left(\Sigma^{0}, \Lambda \pi^{0}\right)=g\left(\Sigma^{-}, \Lambda \pi^{-}\right) \\
& =g\left(\Lambda, \Sigma^{+} \pi^{-}\right)=g\left(\Lambda, \Sigma^{0} \pi^{0}\right)=g\left(\Lambda, \Sigma^{-} \pi^{+}\right)
\end{aligned}
$$

$$
g_{N \Lambda K} \equiv g\left(p, \Lambda K^{+}\right)=g\left(n, \Lambda K^{0}\right)=g\left(\Lambda, p K^{-}\right)=g\left(\Lambda, n \bar{K}^{0}\right),
$$

$$
\begin{aligned}
g_{N \Sigma K} & \equiv g\left(p, \Sigma^{0} K^{+}\right)=-g\left(n, \Sigma^{0} K^{0}\right)=g\left(\Sigma^{0}, p K^{-}\right) \\
= & g\left(\Sigma^{0}, n \bar{K}^{0}\right)=\frac{1}{\sqrt{2}} g\left(p, \Sigma^{+} K^{0}\right)=\frac{1}{\sqrt{2}} g\left(n, \Sigma^{-} K^{+}\right) \\
= & \frac{1}{\sqrt{2}} g\left(\Sigma^{-}, n K^{-}\right)=\frac{1}{\sqrt{2}} g\left(\Sigma^{+}, p \bar{K}^{0}\right), \\
g_{\Lambda} \equiv K & =g\left(\Lambda, \Xi^{0} K^{0}\right)=g\left(\Xi^{0}, \Lambda \bar{K}^{0}\right)=-g\left(\Lambda, \Xi^{-} K^{+}\right) \\
& =-g\left(\Xi^{-}, \Lambda K^{-}\right),
\end{aligned}
$$

$$
g_{N N \eta} \equiv g(n, n \eta)=g(p, p \eta)
$$

For the vector $\rho, \omega$, and $K^{*}$ mesons, the expressions are equivalent to the ones quoted above by making the replacements of Eq. (A11).
[1] E. Jenkins, Nucl. Phys. B375, 561 (1992).

[2] J.F. Dubach, G.B. Feldman, B.R. Holstein, and L. de la Torre, Ann. Phys. (N.Y.) 249, 146 (1996).

[3] A. Parreño, A. Ramos, and C. Bennhold, Phys. Rev. C 56, 339 (1997).

[4] A. Parreño and A. Ramos, Phys. Rev. C (to be published), nucl-th/0104080.

[5] K. Sasaki, T. Inoue, and M. Oka, Nucl. Phys. A669, 331 (2000); A678, 455 (2000).

[6] D.J. Prowse, Phys. Rev. Lett. 17, 782 (1966).

[7] M. Danysz et al., Nucl. Phys. 49, 121 (1963); reanalysis of R.H. Dalitz et al., Proc. R. Soc. London, Ser. A 425, 1 (1989).

[8] S. Aoki et al., Prog. Theor. Phys. 85, 1287 (1991).

[9] Y. Yamamoto, H. Takaki, and K. Ikeda, Prog. Theor. Phys. 86, 867 (1991).

[10] C.B. Dover, D.J. Millener, A. Gal, and D.H. Davis, Phys. Rev. C 44, 1905 (1991).

[11] J. K. Ahn et al., Phys. Rev. Lett. 87, 132504 (2001).

[12] A. Ichikawa et al., Phys. Lett. B 500, 37 (2001).

[13] V.G.J. Stoks and Th.A. Rijken, Phys. Rev. C 59, 3009 (1999); Th.A. Rijken, V.G.J. Stoks, and Y. Yamamoto, ibid. 59, 21 (1999).

[14] A. Ramos, M.J. Vicente-Vacas, and E. Oset, Phys. Rev. C 55, 735 (1997).
[15] H. Outa et al., Nucl. Phys. A670, 281 (2000).

[16] J. Golak, K. Miyagawa, H. Kamada, H. Witala, W. Glöckle, A. Parreño, A. Ramos, and C. Bennhold, Phys. Rev. C 55, 2196 (1997); J. Golak, H. Kamada, K. Miyagawa, H. Witala, and W. Glöckle, Phys. Rev. Lett. 83, 3142 (1999).

[17] J. Caro, C. García-Recio, and J. Nieves, Nucl. Phys. A646, 299 (1999).

[18] B. Holzenkamp, K. Holinde, and J. Speth, Nucl. Phys. A500, 485 (1989).

[19] R. Böckmann, C. Hanhart, O. Krehl, S. Krewald, and J. Speth, Phys. Rev. C 60, 055212 (1999).

[20] A. Parreño, A. Ramos, C. Bennhold, and D. Halderson, in Dynamical Features of Nuclei and Finite Fermi Systems, edited by X. Viñas, M. Pi, and A. Ramos (World Scientific, Singapore, 1994), p. 318.

[21] D. Halderson, Phys. Rev. C 48, 581 (1993).

[22] M.M. Nagels, T.A. Rijken, and J.J. de Swart, Phys. Rev. D 15, 2547 (1977); P.M.M. Maessen, Th. A. Rijken, and J.J. de Swart, Phys. Rev. C 40, 2226 (1989).

[23] L. de la Torre, Ph.D. thesis, University of Massachusetts, 1982.

[24] D. Jido, E. Oset, and J.E. Palomar, Nucl. Phys. A694, 525 (2001).

[25] L. Tiator, C. Bennhold, and S.S. Kamalov, Nucl. Phys. A580, 455 (1994). 
[26] I. Vidaña, A. Polls, A. Ramos, M. Hjorth-Jensen, and V.G.J. Stoks, Phys. Rev. C 61, 025802 (2000).

[27] J. Dabrowski, Phys. Rev. C 60, 025205 (1999).

[28] K. Itonaga et al., in Proceedings of the International Conference on Hypernuclear and Strange Particle Systems [Nucl. Phys. A (to be published)].

[29] K. Itonaga, T. Ueda, and T. Motoba, Nucl. Phys. A585, 331c
(1995).

[30] M.J. Savage and R.P. Springer, Phys. Rev. C 53, 441 (1996); 54, 2786(E) (1996).

[31] R. Springer (private communication).

[32] S. Rinke, diplom-thesis, TU Dresden (2000); M.J. Savage and R.P. Springer, Phys. Rev. C 57, 1478 (1998).

[33] B.R. Holstein (private communication). 